\title{
Prognostic value of splenic volume in hepatocellular carcinoma patients receiving transarterial chemoembolization
}

\author{
Hai-Tao Dai"^, Bin Chen ${ }^{\#}$, Ke-Yu Tang^, Gui-Yuan Zhang, Chun-Yong Wen^, Xian-Hong Xiang, \\ Jian-Yong Yang, Yan Guo, Run Lin^, Yong-Hui Huang^ \\ Department of Radiology, The First Affiliated Hospital, Sun Yat-sen University, Guangzhou, China \\ Contributions: (I) Conception and design: HT Dai, R Lin; (II) Administrative support: YH Huang, Y Guo; (III) Provision of study materials or \\ patients: HT Dai; (IV) Collection and assembly of data: KY Tang; (V) Data analysis and interpretation: B Chen; (VI) Manuscript writing: All authors; \\ (VII) Final approval of manuscript: All authors. \\ \#These authors contributed equally to this work. \\ Correspondence to: Yan Guo; Run Lin; Yong-Hui Huang. No.58, Zhongshan 2nd Road, Guangzhou 510080, China. Email: dr.guoyan@163.com; \\ linrun5@mail.sysu.edu.cn; hyongh@mail.sysu.edu.cn.
}

Background: Liver function is a key determinant for the survival of hepatocellular carcinoma (HCC) patients receiving transarterial chemoembolization (TACE). However, establishing robust prognostic indicators for liver insufficiencies and patient survival remains an unmet demand. This retrospective study evaluated the prognostic value of splenic volume (SV) in HCC patients undergoing TACE.

Methods: A total of $67 \mathrm{HCC}$ patients who underwent at least two consecutive TACE procedures were retrospectively included in this study. Comprehensive clinical information and follow-up data were collected, and the SV was measured based on dynamic contrast enhanced images. Risk factors of SV enlargement were assessed. The prognostic value of SV on survival was analyzed and compared with Child-Pugh (CP) classification and albumin-bilirubin (ALBI) grade.

Results: The baseline SV was $299.74 \pm 143.63 \mathrm{~cm}^{3}$, and showed a moderate and statistically significant correlation with $\mathrm{CP}$ classification $(\mathrm{R}=0.31, \mathrm{P}<0.05)$. The $\mathrm{SV}$ increased remarkably after the first and second TACE procedures $\left(330.16 \pm 155.38 \mathrm{~cm}^{3}, \mathrm{P}<0.01\right.$, and $355.63 \pm 164.26 \mathrm{~cm}^{3}, \mathrm{P}<0.01$, respectively). In survival analysis, the optimal cut-off value of SV was determined as $373 \mathrm{~cm}^{3}$ using X-tile software, and the patients were divided into the small SV group and the large SV groups accordingly. Based on the pre-TACE SV, the median overall survival (mOS) for patients in the small SV group and the large SV group was 458 days and 249 days, respectively $(\mathrm{P}<0.05)$. After the first and second TACE, the mOS in the small SV group and the large SV group were 454 vs. 266 days $(\mathrm{P}<0.05)$ and 526 vs. 266 days $(\mathrm{P}<0.05)$, respectively. No prognostic value of CP classification and ALBI grade was identified for these patients. Furthermore, there were no significant differences between the small and large SV groups in age, tumor stage, and ALBI grade, except for CP classification $(\mathrm{P}<0.05)$.

Conclusions: SV was correlated with CP classification and was a robust predictor for HCC patients undergoing TACE treatment.

Keywords: Hepatocellular carcinoma (HCC); transarterial chemoembolization (TACE); splenic volume (SV); liver function; prognosis, survival

Submitted Apr 02, 2021. Accepted for publication Jun 03, 2021.

doi: 10.21037/jgo-21-226

View this article at: https://dx.doi.org/10.21037/jgo-21-226

^ ORCID: Hai-Tao Dai, 0000-0003-0946-7670; Bin Chen, 0000-0002-5972-8165; Ke-Yu Tang, 0000-0002-9309-4207; Chun-Yong Wen, 0000-0003-0640-7293; Run Lin, 0000-0003-3692-5753; Yong-Hui Huang, 0000-0001-5921-583X. 


\section{Introduction}

Transarterial chemoembolization (TACE) is a recommended treatment with proven therapeutic efficacy and survival benefit for patients with unresectable hepatocellular carcinoma (HCC) (1). As most HCC lesions are developed on a background of cirrhosis of diverse etiologies, liver function status is a key determinant of patient survival in addition to tumor burden (2). In fact, liver dysfunction is the most common cause of post-TACE death (3). Unfortunately, injury of adjacent non-tumor liver is almost inevitable during TACE procedures, which further exacerbates the underlying compensatory capacity that may result in acute or chronic liver failure, and ultimately deteriorates the prognosis $(3,4)$. Furthermore, repeated TACE procedures are frequently required to attain a maximal possible tumor control, particularly for huge lesions. Consequently, to develop an optimal management plan, it is vital to establish robust indicators for predicting liver dysfunction and patient survival following TACE treatment. Currently, liver function is mainly determined by the Child-Pugh classification (CP) or the albumin-bilirubin (ALBI) grade, both of which have certain shortcomings. For instance, CP classification is less consistent and reproducible due to the subjective parameters employed, while ALBI grade suffers from significant intragroup heterogeneity $(5,6)$. Therefore, there is still an urgent need in developing better methods for assessing liver function, and more importantly the prognosis.

The damage of adjacent liver parenchyma results in inflammation, tissue hyperplasia, and fibrogenesis, leading to cirrhosis, elevated portal venous pressure (PVP), and eventually portal hypertension $(\mathrm{PH})$, all of which are proven risk factors of liver dysfunction after TACE treatment (7-9). The spleen is a susceptible organ to these complex pathophysiological alterations and responds quickly and significantly in the form of splenomegaly $(10,11)$. It has been demonstrated that the splenic volume (SV) is closely associated with the severity of cirrhosis and $\mathrm{PH}(12,13)$. In addition, the presence and the magnitude of splenomegaly has been shown to be a reliable predictor for liver failure and overall survival in HCC patients who undergo hepatectomy (11). Therefore, it is reasonable to hypothesize that SV may serve as an efficient biomarker to monitor liver function reserves and predict patient survival during consecutive TACE treatments.

This retrospective study investigated the feasibility and prognostic value of SV in evaluating the liver compensatory capacity and survival in HCC patients undergoing TACE.
Patients were routinely followed up, and clinical data as well as survival information were collected and analyzed. The changes in SV during TACE procedures and its clinical value were evaluated. We present the following article in accordance with the STROBE reporting checklist (available at https://dx.doi.org/10.21037/jgo-21-226).

\section{Methods}

\section{Patients}

This retrospective study was reviewed and approved by the Ethics Committee of the First Affiliated Hospital, Sun Yat-sen University. Data of all HCC patients who received consecutive TACE in the Department of Interventional Radiology of the First Affiliated Hospital of Sun Yat-sen University between April 2015 and August 2017 were reviewed. Patients were excluded from this study if they (I) received other non-curative therapies prior to TACE treatment; (II) had incomplete data due to any cause; (III) presented with a history of splenic arterial/venous embolism or splenectomy; or (IV) presented with other types of malignancies concurrently (Figure 1). All procedures performed in this study involving human participants were in accordance with the Declaration of Helsinki (as revised in 2013). Individual consent for this retrospective analysis was waived.

\section{Treatment and follow-up}

The "on-demand" TACE strategy was adopted according to the overall evaluation, and all TACE treatments were performed with identical procedures and protocols. Briefly, a transfemoral access was established, and a catheter was advanced and placed super-selectively to the tumorfeeding artery under fluoroscopy guidance (Allura Xper FD20, Philips Medical Systems, Eindhoven, Netherlands). Subsequently, embolic agents loaded with chemotherapeutic agents were delivered into tumor masses. Patients were followed up every 3-6 weeks for routine physical examination and evaluation of treatment responses, liver function, and other parameters .

\section{Assessments}

The tumors were staged according to the Barcelona Clinic Liver Cancer (BCLC) standard (14). Liver function was analyzed using the CP classification and the ALBI grade 
$(15,16)$. Image evaluations were implemented before the initial TACE treatment and during each follow-up period. Plain scanning and dynamic contrast-enhanced computed tomography (CT) examinations were carried out on a CT scanner (Aquilion 64, Toshiba Medical System, Tokyo, Japan) using the scanning protocol as follows: tube voltage,

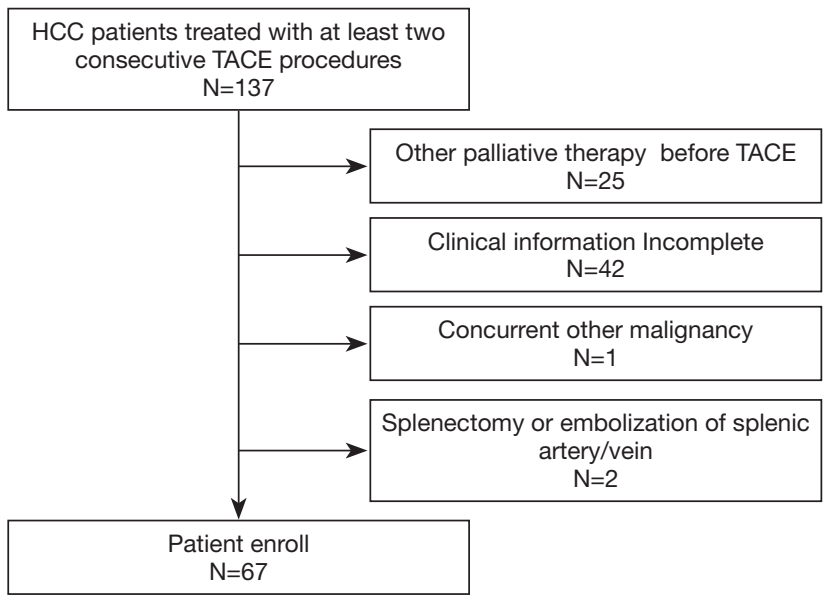

Figure 1 Flowchart of patient enrollment, 67 patients had been enrolled finally. TACE, transarterial chemoembolization; HCC, hepatocellular carcinoma.
$120 \mathrm{kV}$; tube current, $250 \mathrm{mAs}$; rotation time, $0.358 \mathrm{~s}$; field of view, $400 \mathrm{~mm}$; reconstruction interval, $1.0 \mathrm{~mm}$; and slice thickness, $0.8 \mathrm{~mm}$. Image analyses were processed using AnalyzePro 1.0 software (AnalyzeDirect, Overland Park, KS, USA) (17). To determine the SV, radiologist blind to clinical data delineated the spleen manually slide by slide on cross section portal venous phase (PVP) images, and large vessels in spleen were excluded (Figure 2). The volume of the spleen was calculated as the summation of the production of areal and layer thickness automatically using the following formula:

$$
S V=\sum S_{n} \times T_{n}
$$

where $S V$ is the splenic volume, $S$ is the splenic area of each slice, $T$ is the slice thickness, and $n$ is number of slices. Liver function was assessed within 1 week prior to each TACE procedure by the CP classification and ALBI grade (15), and additional tests was carried out when necessary.

In this study, only the data before and after two consecutive TACE treatments were analyzed. This strategy guaranteed better comparability and consistency of the data analysis. The initial TACE treatment aims to achieve a maximal response, and the efficacy and survival benefits of TACE decreases sharply with each subsequent treatment
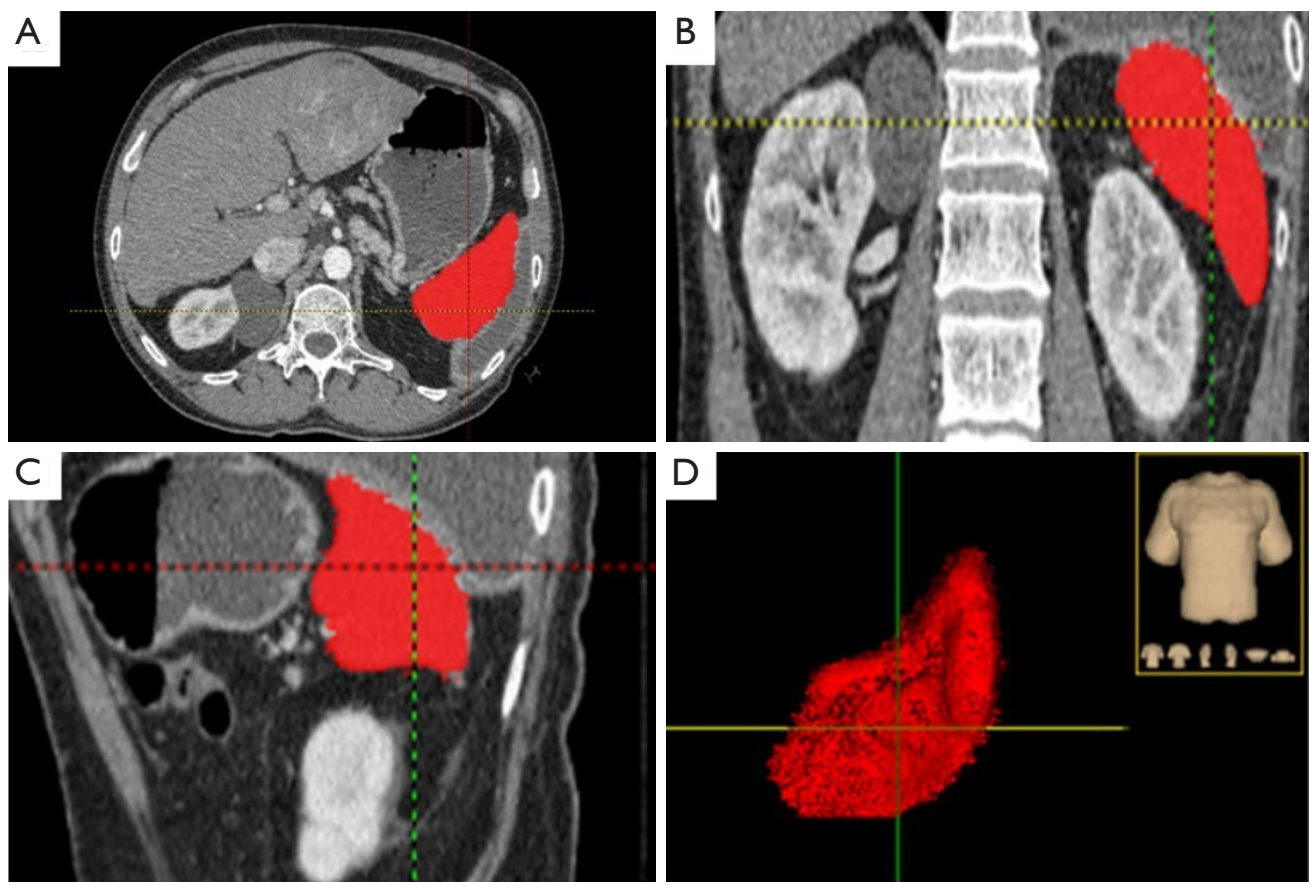

Figure 2 Process of splenic volume measurement. (A) Field of spleen was delineated slide by slide on cross sections. Field of coronal section (B) and sagittal section (C) were accumulated automatically. (D) Tridimensional measurement of splenic volume. 
Table 1 Characteristics of patients enrolled

\begin{tabular}{|c|c|}
\hline Characteristic & Outcome \\
\hline Age (years) & $48.76 \pm 11.07$ \\
\hline \multicolumn{2}{|l|}{ Gender, n (\%) } \\
\hline Male & 63 (94.03) \\
\hline Female & $4(5.97)$ \\
\hline \multicolumn{2}{|l|}{ Etiology, n (\%) } \\
\hline HBV & $67(100.00)$ \\
\hline Other & $0(0)$ \\
\hline \multicolumn{2}{|c|}{ BCLC stage, n (\%) } \\
\hline Stage A & $6(8.96)$ \\
\hline Stage B & 24 (35.82) \\
\hline Stage C & 37 (55.22) \\
\hline \multicolumn{2}{|c|}{ Child-Pugh Class, n (\%) } \\
\hline Class A & $58(86.57)$ \\
\hline Class B & $9(13.43)$ \\
\hline \multicolumn{2}{|c|}{ ALBI Grade, n (\%) } \\
\hline Grade 1 & $17(25.37)$ \\
\hline Grade 2 & 49 (73.13) \\
\hline Grade 3 & $1(1.5)$ \\
\hline Total, n (\%) & $67(100.00)$ \\
\hline
\end{tabular}

HBV, hepatitis B virus; BCLC, Barcelona Clinic Liver Cancer; ALBI, albumin-bilirubin.

attempt (18). If two repeated TACE treatments failed to obtain the expected outcomes, other treatment options were considered, such as tyrosine kinase inhibitors (TKIs) and ablation. To evaluate the prognostic value, the SV at baseline, after the first TACE, and after the second TACE were analyzed. The optimal cutoff value of SV for pre- or post- TACE treatment was calculated using X-tile software (Yale School of Medicine, CT, USA) (19).

\section{Statistical analysis}

Statistical analysis was performed using SPSS 22.0 statistical package (SPSS Inc., Chicago, IL, USA). Continuous data were presented as mean \pm standard deviation $(\mathrm{SD})$ and range. SV before and after TACE was compared using paired $t$-test. Univariate analyses and multivariate analyses were performed to distinguish risk factors. Kaplan-Meier curves and Cox regression models were employed for survival analysis. Categorical variables were compared by Wilcoxon rank sum tests, and the correlation of categorical and consecutive variables were analyzed using the Spearman test. Independent $t$-tests and Chi-square tests were used to evaluate intergroup differences. $\mathrm{P}<0.05$ was considered statistically significant. The optimal cutoff SV value was determined using X-tile software (Yale School of Medicine, CT, USA) (19).

\section{Results}

\section{Patient characteristics}

A total of 67 patients (63 males, 94\%, and 4 females, $6 \%$ ) with an average age of $48.76 \pm 11.07$ years (range, $27-71$ years) were enrolled in this study. The characteristics of the patients are summarized in Table 1. The etiology of the enrolled patients was chronic hepatitis B virus (HBV) infection. No other etiological factors such as hepatitis $\mathrm{C}$ virus $(\mathrm{HCV})$ infections were identified. There were 6 (8.96\%), 24 (35.82\%), and 37 (55.22\%) patients with stage $\mathrm{A}, \mathrm{B}$, and $\mathrm{C}$ tumor, respectively, according to the BCLC staging system. There were $58(86.57 \%)$ and $9(13.43 \%)$ patients with class A and class B CP score, respectively. There were 17 (25.37\%), 49 (73.13\%), and 1 (1.5\%) patient with ALBI grade 1, 2, and 3, respectively.

\section{SV and liver function after repeated TACE}

TACE was successfully implemented in all patients, and no severe or fatal treatment-related complications occurred. The average follow-up duration was $497.12 \pm 417.33$ days. The baseline SV was $299.74 \pm 143.63 \mathrm{~cm}^{3}$. Univariable correlation analysis revealed a moderate and statistically significant correlation between $\mathrm{SV}$ and $\mathrm{CP}$ classification $(\mathrm{R}=0.31, \mathrm{P}=0.011$, Figure $3 A)$. There was also a significant difference in SV between patients of CP class A and class B (Figure 3B). No correlation was found between SV and age, gender, tumor stage, nor ALBI grade at baseline (Figure 3). Furthermore, there was no significant difference in the $\mathrm{SV}$ of patients with different ALBI grade and tumor stage (Figure 3C,D).

The SV increased remarkably after the first TACE procedure $\left(330.16 \pm 155.38 \mathrm{~cm}^{3}, \mathrm{P}<0.01\right)$, and it increased significantly again after the second TACE treatment $\left(355.63 \pm 164.26 \mathrm{~cm}^{3}, \mathrm{P}<0.01\right.$, Figure $\left.4 A\right)$. Subgroup analysis indicated that the SV increased significantly in patients 
A

\begin{tabular}{lll} 
Parameter & R & Sig (P value) \\
\hline Age & -0.17 & 0.165 \\
Sex & -0.114 & 0.358 \\
Stage & 0.046 & 0.715 \\
ALBI grade & 0.12 & 0.33 \\
C-P class & 0.31 & 0.011
\end{tabular}

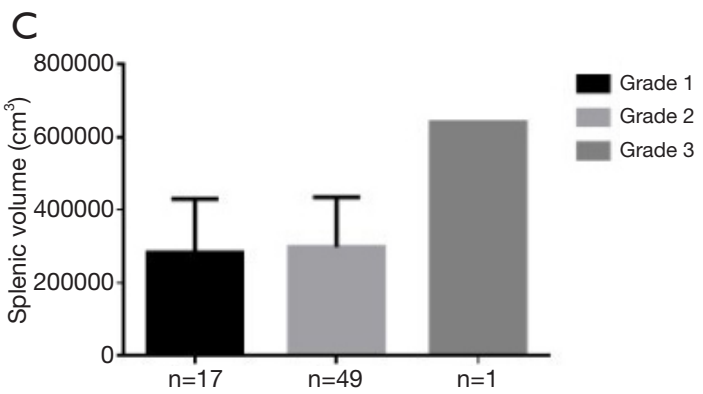

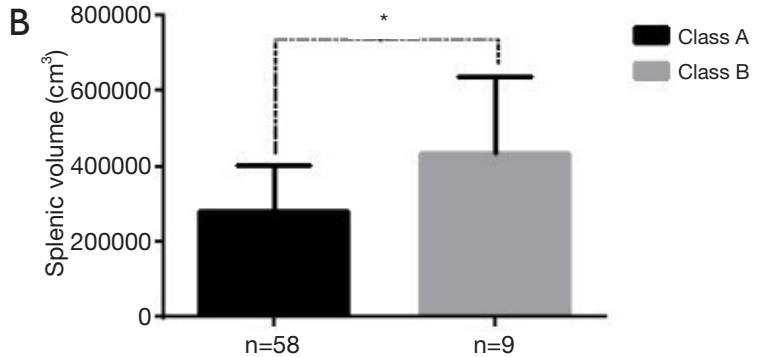

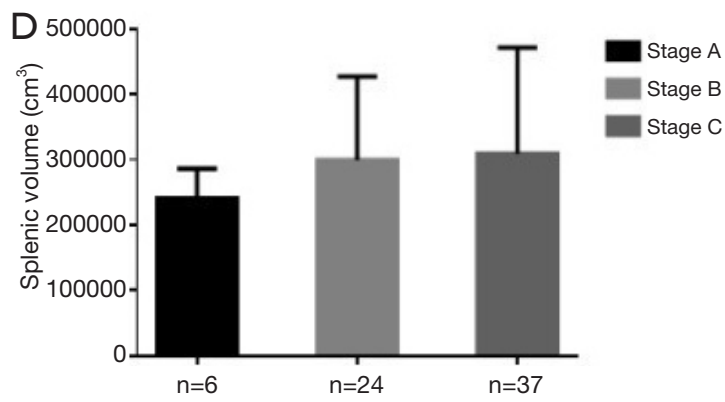

Figure 3 Risk analysis of splenic volume. (A) In univariable analysis, only C-P class showed moderate and significant correlations with basic splenic volume $(\mathrm{P}<0.05)$. (B) Splenic volume was $278.78 \pm 122.10 \mathrm{~cm}^{3}$ and $434.84 \pm 200.66 \mathrm{~cm}^{3}$ in patients of C-P class A and class B, respectively $(\mathrm{P}<0.05)$. (C) Splenic volume was $283.93 \pm 146.30,298.28 \pm 136.84$ and $640.39 \mathrm{~cm}^{3}$ in patients with ALBI grade 1,2 and 3 , respectively $(\mathrm{P}>0.05)$. (D) Splenic volume was $240.43 \pm 45.84,299.47 \pm 127.9$ and $309.54 \pm 162.47 \mathrm{~cm}^{3}$, in patients with tumor stage A, B and C, respectively $(\mathrm{P}>0.05)$. C-P, Child-Pugh; ALBI, albumin-bilirubin; *, significant difference.

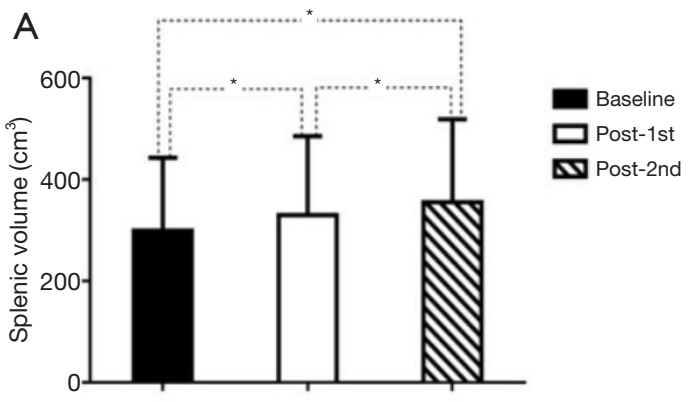

B

\begin{tabular}{lll} 
C Parameter & $\mathbf{R}$ & Sig (P value) \\
\hline Age & -0.213 & 0.083 \\
Gender & 0.114 & 0.358 \\
Tumor Stage & 0.042 & 0.743 \\
Baseline SV & 0.085 & 0.492 \\
C-P Class & -0.007 & 0.957 \\
ALBI Grade & -0.079 & 0.523 \\
Embolic type & -0.075 & 0.391
\end{tabular}

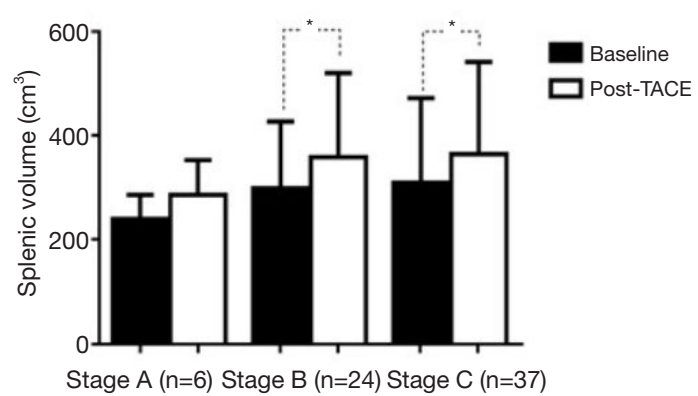

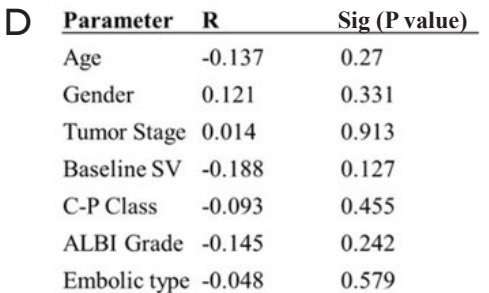

Figure 4 Analysis of splenic volume shift. (A) Splenic volume was $299.74 \pm 143.63 \mathrm{~cm}^{3}$ at baseline, $330.16 \pm 155.38 \mathrm{~cm}^{3}$ after $1 \mathrm{st}$ TACE and $355.63 \pm 164.26 \mathrm{~cm}^{3}$ after $2 \mathrm{nd}$ TACE. Splenic volume enlarged significantly along with each TACE procedure $\left({ }^{*}, \mathrm{P}<0.01\right)$. (B) Splenic volume enlargement was significantly in patients of stage B (absolute value: $59.06 \mathrm{~cm}^{3}, 95 \%$ CI: 28.92-89.20, *, P<0.01) and C (absolute value: $55.45 \mathrm{~cm}^{3}, 95 \%$ CI: 26.98-83.91, *, $\mathrm{P}<0.01$ ), but the enlargement was not obvious in patient of stage A (absolute value: $45.86 \mathrm{~cm}^{3}, 95 \%$ CI: -40.97 to $132.7, \mathrm{P}=0.23)$. Univariable analysis of the absolute enlargement value (C) and relative change rate (D) of splenic volume. TACE, transarterial chemoembolization; SV, splenic volume; C-P, Child-Pugh; ALBI, albumin-bilirubin. 
Table 2 Child-Pugh classification and ALBI grade analysis periTACE. Only ALBI grade showed significant deterioration after 2 TACE procedures $(\mathrm{P}<0.05)$

\begin{tabular}{lcccc}
\hline Criteria & Deteriorate & Stable & Improve & Sig (P value) \\
\hline C-P Class & 13 & 48 & 6 & 0.071 \\
ALBI Grade & 15 & 48 & 4 & 0.012 \\
\hline
\end{tabular}

C-P, Child-Pugh; ALBI, albumin-bilirubin; TACE, transarterial chemoembolization

with BCLC stage B and stage C, but not stage A patients (Figure $4 B$ ). However, univariate analyses showed no significant correlations between the change in SV (either absolute increased volume or relative change ratio) and age, gender, tumor stage, baseline SV, CP classification, ALBI grade, nor type of embolic agent used (lipiodol or microsphere) (Figure 4C,D).

Examination of post-TACE liver function revealed that CP classification in 13 patients and ALBI grade in 15 patients deteriorated after repeated TACE. Wilcoxon rank sum tests demonstrated that only ALBI grade showed significant deterioration after two TACE procedures (Table 2).

\section{Survival analysis}

Kaplan-Meier analysis was performed to assess and compare the prognostic values of SV, CP classification, and ALBI grade (Figure 5). The median overall survival (mOS) in the whole cohort was 424.0 days [ $95 \%$ confidence interval (CI): 297.9 to 550.1]. The optimal cutoff of SV values was determined as $373 \mathrm{~cm}^{3}$ for both pre- and post- TACE treatment (19). The patients were then divided into the small SV group ( $\mathrm{SV}<373 \mathrm{~cm}^{3}$ ) and the large SV group (SV $\geq 373 \mathrm{~cm}^{3}$ ) accordingly. There were 51,47 , and 42 patients in small SV group at baseline, post the first TACE, and post the second TACE, respectively. In the large SV group, there were 16, 20, and 25 patients at baseline, post the first TACE, and post the second TACE, respectively.

The prognostic value of both pre- and post- TACE SV were analyzed. Based on the pre-TACE SV, the mOS was 458 days (95\% CI: 306.40 to 609.60 ) in patients in the small $\mathrm{SV}$ group, which was significantly better than the mOS of 249 days observed with patients in the large SV group (95\% CI: 197.72 to 300.28; $\mathrm{P}<0.05$; Figure 5B). Similarly, according to the SV after the first TACE treatment, mOS in the small SV group and the large SV group were 454 days (95\% CI: 238.23 to 669.70 ) and 266 days (95\% CI: 19.94 to
512.06), respectively ( $\mathrm{P}<0.05$, Figure $5 C)$. Furthermore, for patients who received two TACE procedures, the mOS was 526 days (95\% CI: 359.76 to 692.24 ) in the small SV group compared to 266 days (95\% CI: 226.06 to 305.94 ) in the large $\mathrm{SV}$ group $(\mathrm{P}<0.05$, Figure $5 D)$.

Tumor stage was also presented certain predictive value. There were significant differences in survival for stage A vs. stage $\mathrm{B}$ patients, and stage A vs. stage $\mathrm{C}$ patients, but not stage B vs. stage C patients (Figure $5 E$ ). On the contrary, neither CP classification nor ALBI grade showed predictive values for patient survival (Figure $5 F, G$ ). Likewise, age and gender had no predictive value (Table 3). Subsequent multivariable Cox regression analyses revealed that $\mathrm{SV}$ group [hazard ratio (HR ) 3.07, 95\% CI: 1.42 to 6.67 , $\mathrm{P}=0.004]$ and tumor stage (stage $\mathrm{B} v s$. stage $\mathrm{A}, \mathrm{HR} 4.32$, 95\% CI: 1.21 to $15.41, \mathrm{P}=0.024$; stage $\mathrm{C} v$ s. stage $\mathrm{A}, \mathrm{HR}$ 4.43, 95\% CI: 1.30 to $15.11, \mathrm{P}=0.017$ ) were predictors for survival (Table 4). However, no predictive values were identified for CP classification and ALBI grade.

\section{Comparison of baseline characteristics between the small and large $S V$ groups}

The baseline characteristics between the small and large SV groups were compared. There were 47 CP class A $(92.16 \%)$ and 4 CP class B $(7.84 \%)$ patients in the small SV group, and $11 \mathrm{CP}$ class A (68.75\%) and 5 CP class B (31.25\%) patients in the large $\mathrm{SV}$ group, respectively $(\mathrm{P}<0.05$, Figure $6 A)$. However, there were no significant differences between groups with respect to the other characteristics including ALBI grade (grade 1, $\mathrm{n}=13,25.49 \%$; grade 2, $\mathrm{n}=38,74.51 \%$; and grade 3 , $\mathrm{n}=0,0 \%$ in the small SV group $v$ s. grade $1, \mathrm{n}=4,25 \%$; grade 2 , $\mathrm{n}=11,68.75 \%$; and grade $3, \mathrm{n}=1,6.25 \%$ in the large SV group; $\mathrm{P}=0.31$; Figure $6 B)$, age $(48.92 \pm 11.37$ in the small $\mathrm{SV}$ group and $48.52 \pm 10.36$ in large SV group; $\mathrm{P}=0.83$; Figure $6 C$ ), and tumor stage distribution (stage $\mathrm{A}, \mathrm{n}=6,11.76 \%$; stage $\mathrm{B}, \mathrm{n}=17$, $33.33 \%$; and stage $C, n=28,54.9 \%$ in the small SV group vs. stage $A, n=0,0 \%$; stage $B, n=7,43.75 \%$; and stage $C, n=9$, $26.25 \%$ in the large SV group; $\mathrm{P}=0.39$; Figure $6 D$ ).

\section{Discussion}

Balancing the therapeutic benefits and adverse effects of a candidate treatment option and predicting survival are vitally important for the development of an optimal management plan. This is particularly true in patients who undergo TACE as most of the HCC lesions arise in the background of established cirrhosis with impaired liver 

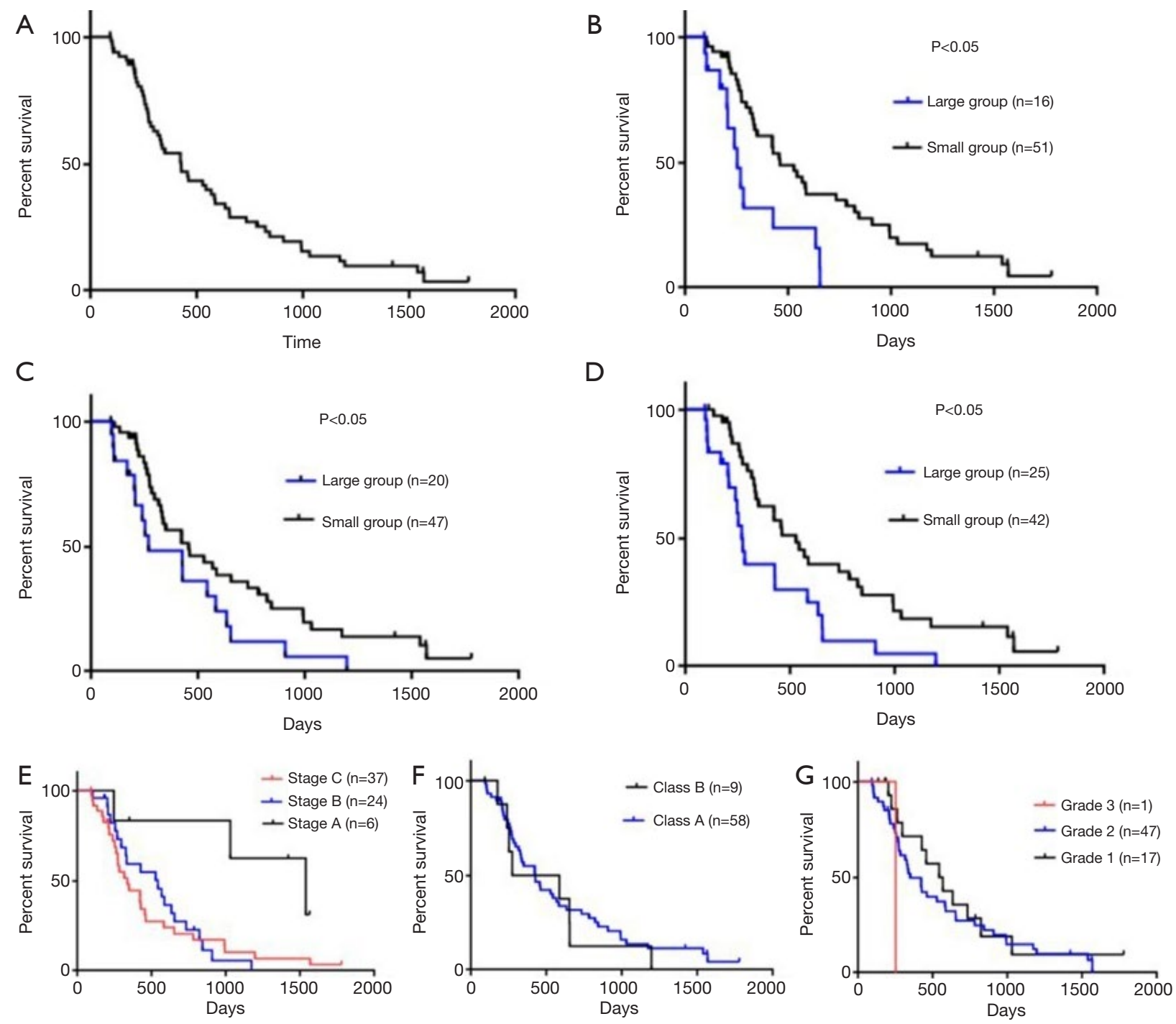

Figure 5 Kaplan-Meier curves of whole cohort (A), in SV groups at baseline (B), post 1st TACE (C) and post 2nd TACE (D), in different tumor stage (E), in different Child-Pugh class (F) and in different ALBI grade (G). SV, splenic volume; TACE, transarterial chemoembolization; ALBI, albumin-bilirubin.

compensatory capacity $(1,5,16)$. When treating tumors, TACE simultaneously causes a series of complex and detrimental pathophysiological changes such as fibrosis, cirrhosis, $\mathrm{PH}$, varices, and even liver failure, which ultimately lead to dismal prognosis $(4,7,9,20)$. Therefore, a robust indicator for evaluating liver function reserve and patient survival is essential to accurately determine whether a patient is suitable for TACE treatment.

In this study, the comprehensive clinical data of 67 consecutive HCC patients who were subjected to repeated TACE procedures were collected to analyze the prognostic value of SV. Clinically used methods, including CP classification and ALBI grade, were adopted for liver function evaluation. Of them, 6 patients in BCLC stage A received TACE mainly due to contradictions of radical treatments. The remaining patients were in BCLC stage B $(n=24)$ or C $(n=37)$ with CP class A or B. Noteworthy, one patient in CP class $\mathrm{B}$ (8 points) with obstructive jaundice secondary to tumor compression of the bile duct was categorized as ALBI grade 3 and underwent TACE in order to reduce tumor burden and to relieve the oppression as soon as possible. Super-selective chemoembolization 
Table 3 Univariable analysis of possible factors with overall survival

\begin{tabular}{lc}
\hline Parameter & Sig (P value) \\
\hline Age & 0.409 \\
Gender & 0.895 \\
Stage A vs. B & 0.004 \\
Stage A vs. C & 0.021 \\
Stage B vs. C & 0.738 \\
C-P class & 0.593 \\
ALBI Grade 1 vs. 2 & 0.398 \\
ALBI Grade 1 vs. 3 & 0.079 \\
ALBI Grade 2 vs. 3 & 0.238 \\
SV group & 0.003 \\
\hline
\end{tabular}

C-P, Child-Pugh; ALBI, albumin-bilirubin; SV, splenic volume.
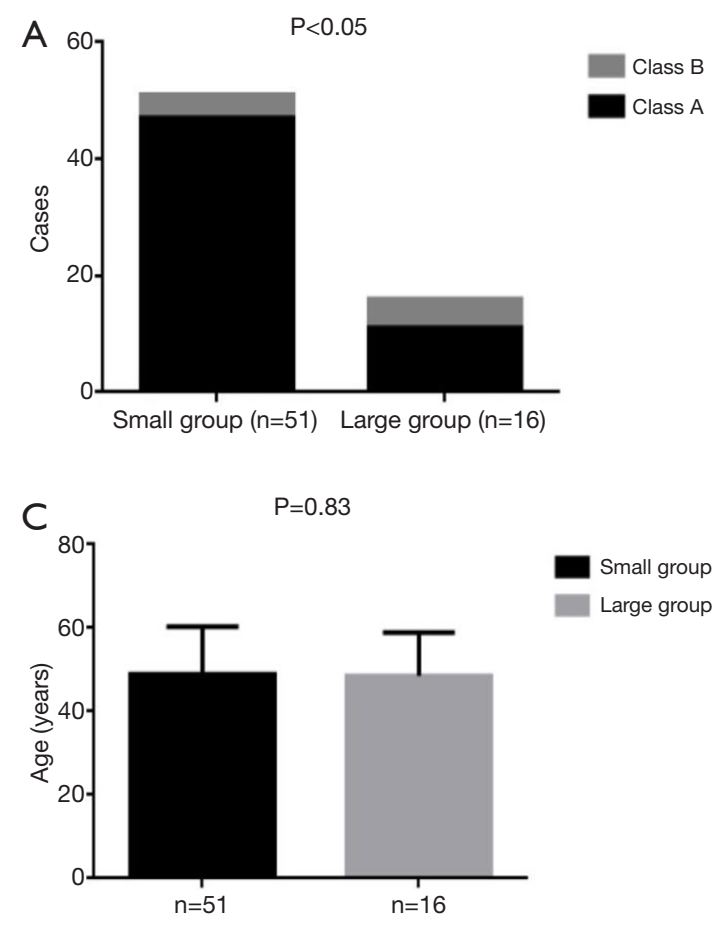

Table 4 Multivariable analysis of risk factors with overall survival

\begin{tabular}{lccc}
\hline Parameter & Sig (P value) & $\mathrm{HR}$ & $95 \% \mathrm{Cl}$ \\
\hline Child-Pugh class & 0.139 & $\mathrm{NA}$ & $\mathrm{NA}$ \\
ALBI & 0.223 & & \\
Grade 2 vs. 1 & 0.223 & $\mathrm{NA}$ & $\mathrm{NA}$ \\
Grade 3 vs. 1 & 0.433 & $\mathrm{NA}$ & $\mathrm{NA}$ \\
Tumor stage & 0.056 & & \\
Stage B vs. A & 0.024 & 4.32 & $1.21-15.41$ \\
Stage C vs. A & 0.017 & 4.43 & $1.30-15.12$ \\
SV group & 0.004 & 3.07 & $1.42-6.67$ \\
\hline ALBl, albumin-bilirubin; SV, splenic volume.
\end{tabular}
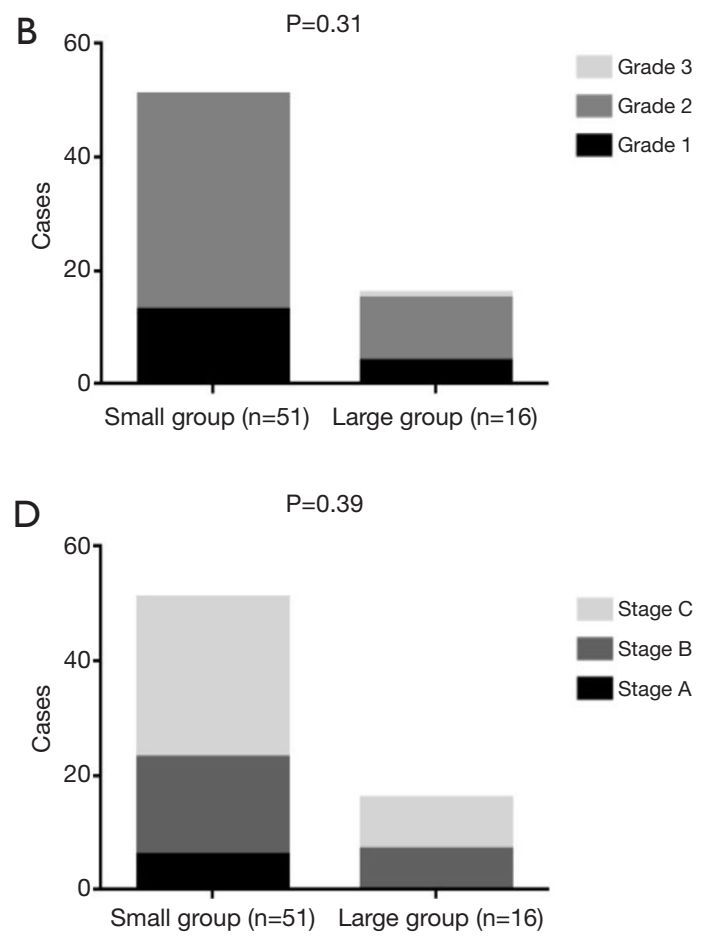

Figure 6 Comparison of characteristics of patients in SV groups. (A) Child-Pugh class: in small group, there were 47 (92.16\%) patients with C-P class A, 4 (7.84\%) patients with C-P class B, while 11 (68.75\%) and 5 (31.25\%) in large group, respectively (P<0.05). (B) ALBI grade in groups: in small group, there were 13 (25.49\%), 38 (74.51\%), 0 (0\%) patients with ALBI grade 1, 2and 3, while 4 (25\%), 11 (68.75\%), $1(6.25 \%)$ in large groups, respectively $(\mathrm{P}=0.31)$. (C) Age of patients in small and large groups showed no difference $(48.92 \pm 11.37$ vs. 48.52 $\pm 10.36, \mathrm{P}=0.83)$. (D) Tumor stage distribution: In small group, there were $6(11.76 \%), 17$ (33.33\%), 28 (54.9\%) patients with tumor burden of stage A, B and C, while 0 (0\%), 7 (43.75\%), 9 (56.25\%) in large group, respectively (P=0.39). SV, splenic volume; C-P, ChildPugh; ALBI, albumin-bilirubin. 
with low-dose chemotherapeutic agent was applied. The tumor regressed and the obstructive jaundice palliated after treatment, and the CP classification and ALBI grade were downgraded from class B and grade 3 to class A and grade 2, respectively.

The baseline and the changes in SV following TACE treatment were explored. The threshold for dividing patients into the small and large SV groups was determined as $373 \mathrm{~cm}^{3}$. The baseline SV showed moderate and statistically significant correlation with CP classification, and there was a significant difference between groups in terms of CP classification, but not other characteristics including age, gender, tumor stages, and ALBI grade. CP classification utilizes clinical symptoms and serum variables to evaluate liver function reserve. It involves not only hepatic metabolic and synthetic function, but also the status of liver fibrosis, cirrhosis, and PH. Similarly, SV is one of the most significant indicators of portal pressure, and it increases in the case of PH. Severe liver fibrosis/cirrhosis and $\mathrm{PH}$ tend to manifest as splenomegaly and higher $\mathrm{CP}$ scores. This is in line with the findings of this study.

The study also demonstrated that SV increased significantly after each TACE procedure, and the increment was an independent process that was not correlated with baseline liver function status, gender, nor tumor stage. Concurrent liver injury is the most common complication of TACE, regardless of the type of embolic agent used $(3,21)$. The mechanisms and consequences of TACEinduced liver injury are complex. In addition to the toxicity of chemotherapeutic agents, TACE also elicits a series of environmental and cytokine signals alterations such as hypoxia induced factor- $1 \alpha(\mathrm{HIF}-1 \alpha)$ and vascular endothelial growth factor (VEGF), which promote fibrosis, cirrhosis, PH, and subsequent splenomegaly $(9,20,22)$. In the survival analysis, both pre- and post-TACE SV exhibited strong predictive power for patient survival, whereas CP score and ALBI grade failed to show prognostic value. CP score is a long-established approach for assessing liver function, and it presents prognostic value in certain clinical scenarios such as surgical resection (15). However, $\mathrm{CP}$ scores bear some limitations which may impair its predictive effect. It comprises subjective indicators, such as the presence and degree of ascites and encephalopathy, which may lead to inconsistent and inaccurate scoring. For example, the severity of ascites is not only determined by liver function. It may also be affected by metastases to the abdominal cavity, vascular occlusion due to tumor invasion and compression, infections, and inflammation, or the combination thereof. Furthermore, evident heterogeneity within the same classification is also an important issue (15). On the other hand, the ALBI grade was developed to improve the effectiveness and accuracy of liver function assessment. It does have a better discriminatory ability than $\mathrm{CP}$ scores, and has been shown to have prognostic value in many studies $(5,15,23)$. However, several drawbacks, such as great heterogeneity, still hamper the wide-spread application of this approach. For instance, patients of ALBI grade 2 may present with a wide range of liver status according to the CP classification, scoring from 5 to 8 or even greater (24). Moreover, serum bilirubin and serum albumin are susceptible to many factors such as hemolysis, inflammation, nutritional condition, or circulation state aside from liver function, and are relatively less specific and sensitive (25).

In contrast, $\mathrm{SV}$ has numerous advantages as a predictive factor. The spleen contains highly elaborate tissue structures and is anatomically linked to the liver via the portal vein system. Clinically, splenomegaly is one of the most important manifestations of liver cirrhosis and PH (26). More importantly, splenomegaly not only responds to, but also participates in the processes of liver fibrosis and hyperplasia (27). Previous studies have revealed that splenomegaly may in turn promote the progression of liver fibrosis to cirrhosis and exacerbate disease prognosis through multiple pathways (28). The size of the spleen has been used to determine the stage of liver fibrosis/cirrhosis and $\mathrm{PH}(13,29)$, and has been identified as an independent predictor of overall survival in patients subjected to adjuvant TACE after surgery (30). Besides, SV is an objective parameter which can be obtained with more accuracy and consistency, and is a consecutive data rather than a discontinuous grade index. The changes in SV, even a slight increment, is easy to identify, which endows SV with dynamic and sensitive predictive power. This is especially easy to be identified in patients receiving TACE as the SV increases significantly after each procedure. However, traditionally, splenomegaly is evaluated by unidimensional proxy long-axis. This method has been demonstrated to be of low sensitivity and specificity, mainly due to the complexity of the shape and orientation of the spleen, and body habitus of diverse populations (31). In this study, the prognostic power was substantially enhanced using threedimensional volumetric measurements and this method was shown to be more precise and reproducible.

There are some limitations in this study. Due to the retrospective study design, numerous patients were excluded 
for certain reasons, and the final sample size was relatively small. In this proof-of-concept study, the SV was calculated based on the manually drawn splenic area layer-by-layer which is somewhat burdensome and time-consuming. Automated or semi-automated methods should be explored in future studies. In addition, future prospective large-scale investigations are warranted to further validate the findings in this study.

\section{Conclusions}

SV exhibited a robust predictive power for HCC patients undergoing TACE treatment and this may be useful in assessing survival benefits versus adverse effects in patients with HCC so as to develop optimal treatment regimens.

\section{Acknowledgments}

Funding: None.

\section{Footnote}

Reporting Checklist: The authors have completed the STROBE reporting checklist. Available at https://dx.doi. org/10.21037/jgo-21-226

Data Sharing Statement: Available at https://dx.doi. org/10.21037/jgo-21-226

Conflicts of Interest: All authors have completed the ICMJE uniform disclosure form (available at https://dx.doi. org/10.21037/jgo-21-226). The authors have no conflicts of interest to declare.

Ethical Statement: The authors are accountable for all aspects of the work in ensuring that questions related to the accuracy or integrity of any part of the work are appropriately investigated and resolved. All procedures performed in this study involving human participants were in accordance with the Declaration of Helsinki (as revised in 2013). This retrospective study was reviewed and approved by the Ethics Committee of the First Affiliated Hospital, Sun Yat-sen University. Individual consent for this retrospective analysis was waived.

Open Access Statement: This is an Open Access article distributed in accordance with the Creative Commons Attribution-NonCommercial-NoDerivs 4.0 International
License (CC BY-NC-ND 4.0), which permits the noncommercial replication and distribution of the article with the strict proviso that no changes or edits are made and the original work is properly cited (including links to both the formal publication through the relevant DOI and the license). See: https://creativecommons.org/licenses/by-nc-nd/4.0/.

\section{References}

1. Villanueva A. Hepatocellular Carcinoma. N Engl J Med 2019;380:1450-62.

2. Yang JD, Kim WR, Coelho R, et al. Cirrhosis is present in most patients with hepatitis B and hepatocellular carcinoma. Clin Gastroenterol Hepatol 2011;9:64-70.

3. Lencioni R, de Baere T, Soulen MC, et al. Lipiodol transarterial chemoembolization for hepatocellular carcinoma: A systematic review of efficacy and safety data. Hepatology 2016;64:106-16.

4. Hiraoka A, Kumada T, Kudo M, et al. Hepatic Function during Repeated TACE Procedures and Prognosis after Introducing Sorafenib in Patients with Unresectable Hepatocellular Carcinoma: Multicenter Analysis. Dig Dis 2017;35:602-10.

5. Pinato DJ, Sharma R, Allara E, et al. The ALBI grade provides objective hepatic reserve estimation across each BCLC stage of hepatocellular carcinoma. J Hepatol 2017;66:338-46.

6. Barzakova ES, Schulze-Hagen M, Zimmermann M, et al. Monitoring Liver Function of Patients Undergoing Transarterial Chemoembolization (TACE) by a 13C Breath Test (LiMAx). Cardiovasc Intervent Radiol 2019;42:1702-8.

7. Kim NH, Lee T, Cho YK, et al. Impact of clinically evident portal hypertension on clinical outcome of patients with hepatocellular carcinoma treated by transarterial chemoembolization. J Gastroenterol Hepatol 2018;33:1397-406.

8. Lin PT, Teng $W$, Jeng $W J$, et al. The incidence and predictors of post transarterial chemoembolization variceal bleeding in hepatocellular carcinoma patients. J Formos Med Assoc 2020;119:635-43.

9. Scheiner B, Ulbrich G, Mandorfer M, et al. Short- and long-term effects of transarterial chemoembolization on portal hypertension in patients with hepatocellular carcinoma. United European Gastroenterol J 2019;7:850-8.

10. Faitot F, Allard MA, Pittau G, et al. Impact of clinically evident portal hypertension on the course of hepatocellular 
carcinoma in patients listed for liver transplantation. Hepatology 2015;62:179-87.

11. Bae JS, Lee DH, Yoo J, et al. Association between spleen volume and the post-hepatectomy liver failure and overall survival of patients with hepatocellular carcinoma after resection. Eur Radiol 2021;31:2461-71.

12. Son JH, Lee SS, Lee Y, et al. Assessment of liver fibrosis severity using computed tomography-based liver and spleen volumetric indices in patients with chronic liver disease. Eur Radiol 2020;30:3486-96.

13. Delahaye J, Bazeries P, Lannes A, et al. Doppler ultrasonography devices, including elastography, allow for accurate diagnosis of severe liver fibrosis. Eur J Radiol 2018;108:133-9.

14. Forner A, Reig ME, de Lope CR, et al. Current strategy for staging and treatment: the BCLC update and future prospects. Semin Liver Dis 2010;30:61-74.

15. Johnson PJ, Berhane S, Kagebayashi C, et al. Assessment of liver function in patients with hepatocellular carcinoma: a new evidence-based approach-the ALBI grade. J Clin Oncol 2015;33:550-8.

16. Llovet JM, Kelley RK, Villanueva A, et al. Hepatocellular carcinoma. Nat Rev Dis Primers 2021;7:6.

17. Ray S, Li M, Koch SP, et al. Seasonal plasticity in the adult somatosensory cortex. Proc Natl Acad Sci U S A 2020;117:32136-44.

18. Kim BK, Kim SU, Kim KA, et al. Complete response at first chemoembolization is still the most robust predictor for favorable outcome in hepatocellular carcinoma. J Hepatol 2015;62:1304-10.

19. Camp RL, Dolled-Filhart M, Rimm DL. X-tile: a new bio-informatics tool for biomarker assessment and outcome-based cut-point optimization. Clin Cancer Res 2004;10:7252-9.

20. Qu K, Yan Z, Wu Y, et al. Transarterial chemoembolization aggravated peritumoral fibrosis via hypoxia-inducible factor-1 $\alpha$ dependent pathway in hepatocellular carcinoma. J Gastroenterol Hepatol 2015;30:925-32.

21. Vogl TJ, Lammer J, Lencioni R, et al. Liver, gastrointestinal, and cardiac toxicity in intermediate hepatocellular carcinoma treated with PRECISION

Cite this article as: Dai HT, Chen B, Tang KY, Zhang GY, Wen CY, Xiang XH, Yang JY, Guo Y, Lin R, Huang YH. Prognostic value of splenic volume in hepatocellular carcinoma patients receiving transarterial chemoembolization. J Gastrointest Oncol 2021;12(3):1141-1151. doi: 10.21037/jgo-21-226
TACE with drug-eluting beads: results from the PRECISION V randomized trial. AJR Am J Roentgenol 2011;197:W562-70.

22. Petrillo M, Patella F, Pesapane F, et al. Hypoxia and tumor angiogenesis in the era of hepatocellular carcinoma transarterial loco-regional treatments. Future Oncol 2018;14:2957-67.

23. Feng D, Wang M, Hu J, et al. Prognostic value of the albumin-bilirubin grade in patients with hepatocellular carcinoma and other liver diseases. Ann Transl Med 2020;8:553.

24. Ogasawara $\mathrm{S}$, Chiba T, Ooka Y, et al. Liver function assessment according to the Albumin-Bilirubin (ALBI) grade in sorafenib-treated patients with advanced hepatocellular carcinoma. Invest New Drugs 2015;33:1257-62.

25. Heimbach JK, Kulik LM, Finn RS, et al. AASLD guidelines for the treatment of hepatocellular carcinoma. Hepatology 2018;67:358-80.

26. Tsochatzis EA, Bosch J, Burroughs AK. Liver cirrhosis. Lancet 2014;383:1749-61.

27. Bolognesi M, Merkel C, Sacerdoti D, et al. Role of spleen enlargement in cirrhosis with portal hypertension. Dig Liver Dis 2002;34:144-50.

28. Li L, Duan M, Chen W, et al. The spleen in liver cirrhosis: revisiting an old enemy with novel targets. J Transl Med 2017;15:111.

29. Abraldes JG, Bureau C, Stefanescu H, et al. Noninvasive tools and risk of clinically significant portal hypertension and varices in compensated cirrhosis: The "Anticipate" study. Hepatology 2016;64:2173-84.

30. Gu J, Zhang X, Cui R, et al. Prognostic predictors for patients with hepatocellular carcinoma receiving adjuvant transcatheter arterial chemoembolization. Eur J Gastroenterol Hepatol 2019;31:836-44.

31. Lui FH, Ma P, Helmy M, et al. Ultrasonographic (US) Two-Dimensional Measurement of Spleen Is Superior to Traditional Length Measurement Alone in Diagnosing Cirrhosis. Dig Dis Sci 2021;66:1335-42.

(English Language Editor: J. Teoh) 